\title{
Efeito do exercício intervalado na capacidade aeróbia, composição corporal e na população obesa: uma revisão baseada em evidências
}

\author{
Diego Augusto Santos Silva \\ Núcleo de Pesquisa em Cineantropometria e Desempenho Humano, Centro de \\ Desportos da Universidade Federal de Santa Catarina, Florianópolis, SC, Brasil
}

\begin{abstract}
Resumo: A revisão objetivou trazer evidências científicas acerca do efeito do exercício intervalado na capacidade aeróbia, composição corporal e na população obesa; analisar as evidências acerca da proporção entre esforço e recuperação e fazer considerações a respeito da prescrição do exercício intervalado para obesos. Realizaram-se buscas nas bases Pubmed, Medline, ISI Web of Knowledge, Ebsco Host e Scopus, de artigos a partir do ano 2000. As evidências demonstraram que o exercício intervalado melhora a capacidade aeróbia, diminui a gordura corporal e ajuda a manter e aumentar a massa corporal magra. Ademais, foi relatado que o intervalo entre as sessões é mais eficiente para a perda de peso se for de maneira ativa. Assim, pessoas obesas podem ser inseridas em um programa de treinamento intervalado como forma de redução de gordura, entretanto cuidados têm que ser tomados na prescrição da intensidade e recuperação do exercício.
\end{abstract}

Palavras-chave: Exercício. Atividade motora. Composição corporal. Obesidade. Tolerância ao exercício.

\section{Interval exercise effect on aerobic capacity, body composition and in the obese population: a review based on evidences}

\begin{abstract}
The present review study had as objectives bring scientific evidences about interval exercise effects on aerobic capacity, body composition and on obese population; analyze evidences about the proportion between exertion and recuperation and make considerations regarding interval exercise prescription for the obese population. There were literature revision on Pubmed, Medline, ISI Web of Knowledge, Ebsco Host and Scopus databases, articles from the year 2000. Evidences demonstrated that interval exercise improves aerobic capacity in athletes and non-athletes, reduces body fat and helps maintaining and increasing fat-free mass. Moreover, studies related that the interval between sessions of interval exercise is more efficient for weight loss if active. Therefore, obese people can be inserted in an interval training program as a way to reduce body fat; however caution must be taken on the prescription of exercise intensity and recuperation.
\end{abstract}

Key Words: Exercise. Motor activity. Body composition. Obesity. Exercise tolerance.

\section{Introdução}

O exercício intervalado ou treinamento intervalado é definido como uma forma de condicionamento físico que intercala períodos curtos, porém regulares, de esforço, com períodos de recuperação dentro de uma mesma sessão de exercício e surgiu como uma alternativa para corridas de longas distâncias, como uma forma de intensificar os treinamentos. Entre as décadas de 30 e 50 este tipo de treinamento começou a ser elaborado e estruturado conforme a distância escolhida, ritmo e duração dos períodos de esforço e recuperação. Nos dias atuais, o treinamento intervalado passou a ser o principal método de treinamento dos esportes ligados à resistência, como, por exemplo, corrida de média e longa distância, natação e ciclismo, além de ser bastante utilizado como forma de treinamento de atletas olímpicos (BILLAT, 2001).
O exercício intervalado pode ser usado para melhorar qualquer um dos três sistemas energéticos, pois o intervalo de recuperação neste tipo de exercício pode ser ativo ou passivo. Ao se priorizar o sistema aeróbio neste tipo de treinamento, aplica-se o método extensivo que é caracterizado por um volume elevado e uma intensidade relativamente baixa. $\mathrm{Em}$ contrapartida, quando o volume é baixo e a intensidade elevada o principal sistema atuante é o anaeróbio (MCARDLE et al. 2003).

Além disso, o exercício intervalado é considerado

um método de treinamento aeróbio que estimula os músculos periféricos sem produzir estresse cardiovascular significativo. Este método utiliza curtos períodos de exercício em alta intensidade, chamados de intervalostrabalho, alternados com exercício de leve intensidade ou nenhum exercício, chamados de intervalos-recuperação, que são repetidos em seqüências dentro de uma série de exercícios. 
Diferentemente do treinamento contínuo, não há um estado estável ("steady-state"), ou seja, não é alcançado um estado de equilíbrio do metabolismo, a partir da realização de um exercício em carga fixa (Moreira, 2008, p. 11).

Classicamente as pesquisa com exercício intervalado foram realizadas com atletas, em busca de melhores rendimentos durante as competições (BILLAT et al. 2000). Com o passar dos anos, alguns pesquisadores extrapolaram os limites do esporte e vêm investigando o efeito do treinamento intervalado em variáveis relacionadas à saúde, como na reabilitação cardiovascular (GEORGIOU et al., 2001), melhora do sistema cardiopulmonar (MAKRIDES et al., 1990), redução do peso corporal (KING et al., 2001), adequação do perfil lipídico (TJØNNA et al., 2009) e como forma de tratamento de pessoas obesas (KAMINSKY; WHALEY, 1993).

Até o presente momento não foi encontrado na literatura uma revisão com os principais achados do efeito do treinamento intervalado em variáveis relacionadas à saúde. $\mathrm{O}$ que vem sendo relatado nas bases cientificas são revisões abordando somente uma temática por vez e que não necessariamente tratam somente do exercício intervalado, mas também de outras formas de exercício (BILLAT, 2001; MEYER, 2001). Neste sentido, uma pesquisa que considere evidências científicas de somente um modo de exercício torna-se necessária para esclarecer em qual nível se encontram as investigações relacionadas à temática e quais os principais desafios que precisam ser encarados pela área.

Sendo assim, o presente estudo tem como objetivo trazer evidências científicas acerca do efeito do exercício intervalado na capacidade aeróbia, composição corporal e na população obesa; analisar as evidências acerca da proporção entre esforço e recuperação neste exercício e fazer considerações sobre a prescrição do exercício intervalado para a população com obesidade.

\section{Métodos}

Para a realização da presente revisão, todas as referências foram retiradas das bases de dados Pubmed, Medline, ISI Web of Knowledge, Ebsco Host e Scopus, disponíveis na internet. Nessa perspectiva, foram buscados primeiramente artigos publicados a partir do ano 2000. Entretanto, estudos clássicos que alertaram para a temática foram desenvolvidos no final da década de 80 e inicio de 90 e incluídos na presente pesquisa. Como critério, foram incluídos apenas artigos científicos, os quais deveriam ter a amostra de adultos e/ou idosos e ser na língua inglesa. Para isso, palavras-chave como "Exercises", "Physical Exercise", "Aerobic Exercises", "Interval Exercise", "Interval Training", "Obesity", "Body Composition", foram combinadas de diversas maneiras.

\section{Exercício Intervalado e Capacidade Aeróbia}

Estudos demonstraram que 0 exercício intervalado é uma maneira efetiva de aumentar a capacidade aeróbia de atletas de todos os níveis (BILLAT, 2001; BILLAT et al., 2000).

BILLAT et al. (2000) conduziram um estudo com oito atletas de ciclismo treinados, comparando exercícios de ritmo constante ao treino intervalado. Os participantes realizaram uma sessão de treino em ritmo contínuo a aproximadamente $90 \%$ da velocidade associada ao $\mathrm{IVO}_{2 \operatorname{máx}}$ (velocidade ou potencia na qual o $\mathrm{VO}_{2 m a ́ x}$ é atingido durante um teste incremental), e uma sessão de exercício intervalado a $100 \%$ do $\mathrm{IVO}_{2 \text { máx }}$ por 30 segundos, seguido de 30 segundos a $50 \%$ do $\mathrm{IVO}_{2 \text { máx. }}$ Os resultados mostraram que o exercício intervalado permitiu que os participantes mantivessem seu $\mathrm{VO}_{2 \text { máx }}$ por um período mais longo de tempo, o que resultou em um menor acúmulo de lactato sanguíneo. Os autores do estudo supracitado sugeriram que isso ocorreu devido à mediação do lactato sanguíneo durante os períodos de descanso, que consequentemente, permitiu que 0 atleta se exercitasse por mais tempo.

Amostras de não atletas também foram investigadas utilizando o exercício intervalado como maneira de melhorar a aptidão física (MAKRIDES et al., 1990; SÖKMEN et al., 2002). Um estudo realizado por Makrides et al. (1990) investigaram a eficácia de um programa de 12 semanas de treino intervalado em 12 jovens sedentários e 12 idosos sedentários, todos do sexo masculino. O protocolo utilizado nesse estudo consistiu em sessões de 60 minutos, realizadas três dias por semana com intervalos a $65 \%$ e $45 \%$ do $\mathrm{VO}_{2 \text { pico }}$ (consumo de oxigênio quando o sujeito não mais consegue manter a intensidade determinada durante 0 teste de esforço cardiopulmonar), em uma proporção de um minuto cada, com um aumento gradual na intensidade conforme o passar das semanas. Ao final da intervenção, os idosos apresentaram um aumento de $38 \%$ no $\mathrm{VO}_{2 \text { pico }}(1,60 \pm 0,073$ para $2,21 \pm 0,073$ I.min-1), enquanto os jovens apresentaram uma melhora de $29 \%$ no $\mathrm{VO}_{2 \text { pico }}$ $(2,54 \pm 0,141$ para $3,26 \pm 0,181$ I.min-1). Essa melhora foi também associada a uma melhora na condutância vascular, no rendimento cardíaco máximo, e na capacidade aeróbia muscular. 
Outro estudo conduzido por Sökmen et al. (2002) comparou os efeitos de um típico programa de exercícios de ritmo constante aos efeitos de um programa intervalado de alta intensidade em jovens adultos. Um total de 42 participantes foi dividido aleatoriamente entre os dois grupos, e se exercitaram três vezes por semana por um período de 10 semanas, sendo que a intensidade do exercício foi aumentando com o passar da intervenção. O grupo de ritmo constante se exercitou a uma intensidade constante durante toda a sessão de exercício, enquanto 0 grupo intervalado realizou os exercícios a $120-150 \%$ do $\mathrm{VO}_{2 \text { máx }}$ durante $\mathrm{O}$ período de esforço, e a $30-40 \%$ do $\mathrm{VO}_{2 \text { máx }}$ durante os intervalos de recuperação, dentro de cada sessão. Ambos os grupos apresentaram aumentos significativos no $\mathrm{VO}_{2 \text { máx }}$ e nos tempos de corrida, no entanto, as melhoras foram maiores no grupo de exercícios intervalados. Sökmen et al. (2002) sugeriram que as melhoras na aptidão física tenham sido devidas ao maior esforço aplicado no sistema cardiovascular pelo componente de alta intensidade, empregado durante o exercício intervalado. Além disso, foi verificado que o grupo de treino intervalado também teve uma melhora significativa em testes isocinéticos de extensão e flexão de pernas, enquanto o grupo de ritmo constante não obteve melhora em nenhum teste. Desta forma, pode-se especular que essa melhora no grupo de exercício intervalado tenha sido resultado de um aumento na massa muscular.

Pesquisa desenvolvida por Adeniran e Toriola (1988), investigou o emprego de exercícios intervalados em 76 estudantes do sexo feminino saudáveis, que se exercitaram ao ar livre, três vezes por semana, durante dois meses. Os exercícios consistiam em uma corrida em ritmo constante a $80-85 \%$ da $\mathrm{FC}_{\max }$ (Frequencia cardíaca máxima); ou em exercícios intervalados praticados a $90 \%$ da $\mathrm{FC}_{\text {máx }}$ por quatro minutos, intercalados com quatro minutos de caminhada relaxada, repetidos por quatro vezes durante a sessão. Os resultados indicaram que ambos os grupos de treino tiveram um aumento no seu $\mathrm{VO}_{2 m a ́ x}$, no entanto, o grupo de exercícios intervalados mostrou um aumento ligeiramente maior nas capacidades aeróbia (11,5\%) e anaeróbia $(14,6 \%)$, do que o grupo de ritmo constante (10,2\% e $13,2 \%$, respectivamente). Os autores destacaram que esse aumento foi provavelmente devido à maior carga aplicada ao sistema cardiovascular pelos componentes de alta intensidade, no caso do programa de exercícios intervalados.

Outro estudo realizado por Perry et al. (1986) investigou um programa de dez semanas que consistiu em exercícios intervalados realizados a $85 \%$ da $\mathrm{FC}_{\text {máx }}$, seguidos de descanso (de três em três minutos em um período de 36 minutos). Os participantes incluíam seis homens sedentários e seis mulheres sedentárias no período pósmenopausa. Os resultados mostraram uma queda significativa nas pressões arteriais sistólica (PAS; $127 \pm 4,92$ para $117 \pm 2,49 \mathrm{mmHg}$ ) e diastólica (PAD; 82,1 $\pm 2,66$ para 75,16 $\pm 2,08 \mathrm{mmHg}$ ), e na porcentagem de gordura corporal $(27,55 \pm 1,57$ para $25,75 \pm 1,75 \%$ ), assim como uma melhora no $\mathrm{VO}_{2 \text { máx }}(32,85 \pm 3,00$ para $38,05 \pm 3,23 \mathrm{ml} / \mathrm{kg}$ $1 /$ min-1). Por outro lado, o grupo controle não apresentou nenhuma melhora nessas medidas. Além disso, o grupo de exercícios apresentou uma queda de $6 \%$ no colesterol total, de $11 \%$ nas lipoproteínas de baixa densidade (LDL), de $33 \%$ nas lipoproteínas de muito baixa densidade (VLDL), de $28 \%$ nos níveis de triglicérides, e de $3 \%$ nas lipoproteínas de alta densidade (HDL). O mais importante foi que o exercício intervalado diminuiu significantemente a razão de risco das lipoproteínas (LDL/HDL). Os pesquisadores concluíram que os indivíduos que possuíam os perfis lipídicos menos favoráveis, apresentaram as mudanças mais benéficas (PERRY et al., 1986).

Ahmaidi et al., (1998) também estudaram as respostas clínicas e cardiorrespiratórias aos exercícios intervalados em 22 idosos sedentários. Os indivíduos foram divididos aleatoriamente entre um grupo de trein $\mathrm{o}$ e um controle. $\mathrm{O}$ grupo de treino participou de exercícios intervalados de caminhada/corrida, duas vezes por semana, por doze semanas, nos limiares ventilatórios individuais. Diferentes proporções de exercícios de alta e baixa intensidade foram usadas durante as sessões, assim como diferentes durações de intervalo por sessão, sendo que houve um aumento de 30-60 minutos na duração até a $12^{a}$ semana. Os resultados indicaram um aumento significativo no $\mathrm{VO}_{2 m a ́ x}$ $(1,77 \pm 0,2$ para $2,11 \pm 0,3 \mathrm{~L} / \mathrm{min})$, no limiar ventilatório e na tolerância ao exercício dentro do grupo de treino. É importante mencionar que o programa de exercícios intervalados foi clinicamente bem tolerado por essa população, ao mesmo tempo em que a aderência ao exercício foi excelente (73\%) (AHMAIDI et al., 1998).

Outros benefícios foram associados ao uso de apenas exercícios de alta intensidade. Hardman (2001) conduziu uma meta-análise com o objetivo de investigar os efeitos de exercícios de alta intensidade comparados aos de baixa intensidade, com gasto energético equivalente, na aptidão aeróbia de indivíduos sedentários. Apesar desses estudos não envolveram 
exercícios intervalados, os efeitos dos exercícios de alta intensidade são relevantes, já que podem ser aplicados aos componentes de alta intensidade do treino intervalado. Tais estudos demonstraram que 45-50 minutos de exercício, realizados de três a cinco vezes por semana em alta intensidade elevaram $\circ \mathrm{VO}_{2 \text { máx }}$ a níveis maiores quando comparado a exercícios de intensidade moderada. Um estudo controlado randomicamente relatou uma melhora de $16 \%$ no $\mathrm{VO}_{2 \text { máx }}$ decorrente de exercícios de alta intensidade, contra uma melhora de apenas $4 \%$ diante de intensidades mais baixas (DUNCAN et al., 1991). Além disso, O'Donovan et al. (2005) conduziram uma intervenção de 24 semanas envolvendo homens sedentários, com idades entre 30 e 45 anos, que foram divididos aleatoriamente em três grupos: um grupo controle, um grupo de exercícios de intensidade moderada que se exercitou a $60 \%$ do $\mathrm{VO}_{2 \text { máx }}$, e um grupo de exercícios de alta intensidade que se exercitou a $80 \%$ do $\mathrm{VO}_{2 \text { máx }}$; sendo que nenhum dos grupos realizou alterações na dieta, ou no estilo de vida. Na oitava semana, os dois grupos de exercício estavam participando de três sessões de exercício por semana, os dois com o mesmo gasto calórico, 400 kcals por sessão. Ao final da intervenção, o grupo de exercícios de alta intensidade apresentou um aumento significativo no $\mathrm{VO}_{2 m a ́ x}$, quando comparado ao grupo de intensidade moderada. Por fim, a avaliação do perfil lipídico demonstrou que os fatores de risco coronariano diminuíram apenas no grupo de exercícios de alta intensidade. Assim, o estudo de O'Donovan et al. (2005) concluiu que o exercício de alta intensidade foi uma intervenção mais efetiva para melhorar a condição cardiorrespiratória de homens sedentários, do que o exercício de intensidade moderada, apesar de o custo energético dos exercícios ter sido o mesmo.

\section{Exercício Intervalado e Composição Corporal}

A literatura revelou que o exercício intervalado tem uma influência positiva também na composição corporal. Essa influência foi demonstrada em um estudo realizado por Tremblay et al. (1994), o qual solicitou que 27 indivíduos inativos de peso normal pedalassem por 20 semanas a $60-85 \%$ da FCRes (freqüência cardíaca de reserva), ou participassem de um treino intervalado por 15 semanas, com exercícios de diferentes intensidades e durações durante todo o período de intervenção. Os resultados demonstraram um gasto maior de energia no grupo de ciclistas $(120,4 \pm 31,0 \mathrm{MJ})$ em comparação ao grupo de treino intervalado $(57,9$ $\pm 14,4 \mathrm{MJ})$. Entretanto, é importante mencionar que o grupo de treino intervalado apresentou uma maior mudança nas medidas de dobras cutâneas $(94,2 \pm 37,7$ para $80,3 \pm 36,0 \mathrm{~mm})$ em comparação ao grupo de ciclistas $(79,2 \pm 35,1$ to $74,7 \pm 34,2 \mathrm{~mm}$ ). Quando a soma das dobras cutâneas foi dividida pelo gasto de energia, a quantidade de gordura subcutânea perdida pelo grupo intervalado foi nove vezes maior do que a do grupo de ciclistas, 0 que sugere que 0 exercício de alta intensidade tem um papel ativo na perda de gordura. Os pesquisadores concluíram que os componentes de alta intensidade do exercício intervalado estimularam a utilização pós-exercício de lipídios, e, portanto, favoreceram um déficit de gordura maior após o exercício (TREMBLAY et al., 1994).

Outro estudo realizado por Treuth et al. (1996) comparou 60 minutos de treino de baixa intensidade realizado a $50 \%$ do $\mathrm{VO}_{2 m a ́ x}$, a um treino intervalado com 15 sessões de exercícios praticados a $100 \%$ do $V_{O_{2 m a ́ x}}$, com uma proporção de esforço/recuperação de 2:2 minutos. O grupo de exercício intervalado apresentou maior gasto de energia no período de 24 horas após o exercício, o que resultou em uma maior perda de massa gorda.

O estudo realizado por O'Donovan et al. (2005), mencionado anteriormente, também analisou a composição corporal. A porcentagem de gordura corporal diminuiu significantemente em ambos os grupos de exercício $(22,6 \pm 4,2 \%$ para $22,3 \pm 4,5 \%$ e $23,4 \pm 3,9 \%$ para $21,9 \pm 3,9 \%$ no grupo de exercícios moderados e de alta intensidade, respectivamente), enquanto o grupo controle ganhou gordura corporal durante 0 período de intervenção.

Além de ser um sistema de treino benéfico para atletas treinados, o exercício intervalado pode também ser usado por aqueles que têm dificuldade em manter uma intensidade moderada de exercícios durante uma sessão contínua, devido, por exemplo, à aptidão física muito baixa, ou a doenças crônicas (HUNTER et al., 1998; KAMINSKY; WHALEY, 1993; KING et al., 2002; KING et al., 2001). O exercício intervalado pode ser adaptado para uso em populações obesas, ainda que, até o momento, poucas pesquisas tenham sido realizadas com 0 intuito de determinar a eficácia do exercício intervalado nesse tipo de população.

Um estudo realizado por Kaminsky e Whaley (1993) avaliou o efeito do exercício intervalado no EPOC (consumo de oxigênio excessivo pósexercício) de cinco mulheres obesas e cinco mulheres com peso normal, após dois testes de exercício na esteira ergométrica. O primeiro teste 
consistiu em uma caminhada em ritmo contínuo a $60 \%$ do $\mathrm{VO}_{2 \text { máx }}$, enquanto o segundo envolveu exercícios intervalados realizados em velocidades equivalentes a 30 e $90 \%$ do $\mathrm{VO}_{2 \text { máx }}$ individual. Os resultados demonstraram que 0 EPOC do exercício intervalado foi $127 \%$ mais longo, e $93 \%$ maior em dimensão do que o EPOC da caminhada em ritmo contínuo. Ademais, um consumo de oxigênio excessivo pós-exercício mais longo resulta em um maior gasto de calorias, e deve promover maior perda de peso se a ingestão de calorias for reduzida ou mantida constante.

Além disso, uma investigação feita por King et al. (2002) verificou mudanças na utilização de substratos, comparando um programa de oito semanas de exercícios intervalados a um de exercícios de ritmo constante em mulheres obesas, em período pré-menopausa, sem dieta. As integrantes participaram de três sessões de exercício por semana, com um gasto de $300 \mathrm{kcal}$ por sessão. O programa de exercícios intervalados consistiu em dois minutos de exercício praticados a $95 \%$ do $\mathrm{VO}_{2 \text { máx }}$, seguido por três minutos a $25 \%$ do $\mathrm{VO}_{2 \text { máx }}$, enquanto 0 programa de ritmo constante, usou uma intensidade constante de $50 \%$ do $\mathrm{VO}_{2 \text { máx. Ambos }}$ os programas foram equivalentes em termos de intensidade média, duração e gasto de calorias. $O$ resultado demonstrou que o exercício intervalado resultou em um aumento significativo no gasto de calorias a um dado valor de taxa de troca respiratória, o que consequentemente aumentou a utilização absoluta de gordura, resultando na perda de gordura. Além disso, $\mathrm{O} \quad \mathrm{VO}_{2 \max }$ aumentou significantemente depois do programa de exercícios intervalados, de 13,59 \pm 3,85 para $16,53 \pm 2,70 \mathrm{ml} / \mathrm{kg}-1 / \mathrm{min}-1$.

Em outra investigação os resultados do grupo de exercício intervalado apresentaram um aumento de $13,1 \%$ no $\mathrm{VO}_{2 \text { máx }}(25.2 \pm 4.2 \mathrm{ml} / \mathrm{kg}$ $1 / \mathrm{min}-1$ para $28.5 \pm 5.1 \mathrm{ml} / \mathrm{kg}-1 / \mathrm{min}-1)$; um aumento de $5,4 \%$ na taxa metabólica de repouso avaliada 24 horas depois do exercício $(1671 \pm 406$ $\mathrm{kcal} /$ dia para $1761 \pm 412 \mathrm{kcal} / \mathrm{dia}$ ); e uma queda de $4,8 \%$ no percentual de gordura corporal ( $45 \pm$ $8.4 \%$ para $42.8 \pm 7.2 \%$ ). O grupo de exercícios com ritmo constante não apresentou nenhuma melhora nessas variáveis após o período de intervenção (KING et al., 2001).

Os estudos citados sugerem que o exercício intervalado seja uma intervenção mais eficiente do que os exercícios aeróbios de ritmo constante em populações com obesidade. A mudança frequente de ritmo do exercício intervalado pode também tornar essa forma de exercício mais interessante de se participar do que uma longa sessão de exercício aeróbio de ritmo constante, o que, consequentemente, pode contribuir para uma maior aderência ao exercício. Além disso, o exercício intervalado pode resultar em um gasto maior de energia como resultado de um EPOC maior e mais longo. De acordo com Hunter et al. (1998), o desenvolvimento de programas eficientes em termos de tempo, e que aumentem as taxas metabólicas, é vital para deter a crescente prevalência da obesidade. Consequentemente, estudos adicionais são necessários para que se possa investigar o impacto do exercício intervalado na perda de peso e na aptidão física das populações obesas.

\section{Exercício Intervalado: Proporção entre Esforço e Recuperação}

Antes de começar um programa de exercícios intervalados é importante determinar a duração mais adequada dos intervalos de esforço/recuperação, para obtenção de resultados mais efetivos decorrentes do treinamento (BILLAT et al., 2001).

Franch et al. (1998) estudaram corredores amadores moderadamente treinados por um período de seis semanas. Os três tipos de exercício utilizados nesse estudo foram: corrida em ritmo contínuo praticada a $90 \%$ do $\mathrm{VO}_{2 \text { máx }}$, exercícios intervalados longos (4:2 minutos de esforço/recuperação) e exercícios intervalados curtos (15:15 segundos de esforço/recuperação) praticados a níveis superiores de $\mathrm{VO}_{2 \text { máx }}$ Os pesquisadores descobriram que as maiores evoluções na aptidão aeróbia foram associadas ao exercício intervalado longo, que gerou uma melhora de $6,0 \%$ no $\mathrm{VO}_{2 \text { máx }}(4,22$ para 4,47 L.min1), comparado a $5,9 \%$ (3,89 para 4,12 L.min-1) e 3,6\% (4,10 para 4,24 L.min-1) dos exercícios em ritmo contínuo e os exercícios intervalados curtos, respectivamente.

Millet et al. (2003) estudaram oito triatletas bem treinados que se exercitaram a $100 \%$ e $50 \%$ da $\mathrm{IVO}_{2 \text { máx. }}$ Três intervenções diferentes foram comparadas, incluindo exercícios com intervalos de esforço/recuperação em uma proporção de 3030 segundos, 60-30 segundos, ou a metade do tempo de exaustão da $I \mathrm{O}_{2 \max }(1 / 2 \mathrm{Tlim})$. Os resultados demonstraram que sessões de corrida intermitentes com o mesmo tempo total, mas com diferentes intervalos de esforço, levaram a diferentes respostas individuais no $\mathrm{VO}_{2}$ e na $\mathrm{FC}$ de atletas. Os valores de pico de consumo de oxigênio foram significantemente mais baixos no grupo de $30-30$ segundos $(65,4 \pm 4,9 \mathrm{ml} / \mathrm{kg}-1 / \mathrm{min}-$ 1) do que nos outros dois grupos (grupo de 60-30 segundos: $71,7 \pm 4,8 \mathrm{ml} / \mathrm{kg}-1 / \mathrm{min}-1$, e $1 / 2 \mathrm{Tlim}$ : $72,2 \pm 6,1 \mathrm{ml} / \mathrm{kg}-1 / \mathrm{min}-1)$. Essa tendência foi 
verificada da mesma maneira nos valores de pico de FC, o grupo de 30-30 segundos também demonstrou valores significantemente mais baixos $(183,1 \pm 8,5 \mathrm{bpm})$ do que os outros dois grupos (grupo de 60-30 segundos: 188,8 \pm 10,4 bpm, e 1/2Tlim: 187,0 $\pm 11,4$ bpm). Esse estudo sustenta o uso de corridas intermitentes de longo intervalo. Além disso, após revisar diversos artigos, Billat (2001) concluiu que a recuperação ativa no exercício intervalado tem vários benefícios em relação à recuperação passiva, já que além de atingir e manter o $\mathrm{VO}_{2 \text { máx }}$, estimula a remoção de lactato.

Em relação a populações obesas e exercício intervalado pôde-se observar poucas investigações, deste modo, a proporção ideal entre os intervalos de esforço e recuperação ainda é desconhecida. Assim, estudos adicionais são necessários para explorar esse conceito, especialmente em relação à composição corporal.

\section{Considerações na prescrição do exercício intervalado para obesos}

Um programa de exercícios intervalados feito sob medida para cada indivíduo, que combine períodos de exercício de baixa intensidade que possam ser praticados em um ritmo confortável de caminhada, e exercícios de alta intensidade em ritmo de caminhada ou corrida pode ser apropriado para populações obesas. Algumas considerações na prescrição do exercício intervalado para obesos necessitam ser seguidas para a melhor aderência do individuo ao programa e para a eficácia do treinamento. Dentre estas considerações pode-se listar:

$>$ Os períodos de exercício de alta intensidade deverão sobrecarregar o sistema energético anaeróbio, encorajando a adaptação, já que são caracterizados pela melhora da aptidão aeróbia e anaeróbia. Consequentemente, o exercício intervalado deve aumentar o consumo máximo de

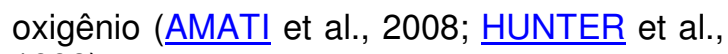
1998);

$>$ Os períodos de exercício de baixa intensidade propiciam a recuperação parcial do exercício de alta intensidade, já que ocorre uma diminuição na frequência cardíaca. Além disso, as reservas de oxigênio, trifosfato de adenosina, e fosfocreatina são parcialmente reabastecidas, e pode haver uma grande remoção de ácido lático (WILLARDSON et al., 2008). Esse processo tende a aumentar a duração total do exercício praticado por um indivíduo, em comparação ao que teria sido alcançado se fossem praticados apenas exercícios de alta intensidade, o que resulta em uma maior queima geral de calorias (QUINN et al., 1994);
$>$ Em comparação ao exercício de baixa intensidade praticado em ritmo constante, o intervalo do exercício de alta intensidade deve resultar em uma temperatura corporal mais alta, em níveis mais altos de ativação hormonal, em um aumento na produção de ácido lático, e em uma maior depleção dos estoques de oxigênio, o que, por fim, resulta em um aumento no tempo de recuperação acumulado, ou EPOC (KAMINSKY; WHALEY, 1993). EPOCs mais longos pós-exercícios de alta intensidade foram relatados por diversos pesquisadores (BØRSHEIM; BAHR, 2003; KAMINSKY; WHALEY, 1993; SHORT; SEDLOCK, 1997; QUINN et al., 1994; TREUTH et al., 1996). Kaminsky e Whaley (1993), mais especificamente, relataram um EPOC mais alto após exercícios intervalados do que após exercícios de ritmo contínuo. Como o EPOC é associado a uma taxa metabólica elevada (BØRSHEIM; BAHR, 2003; SHORT; SEDLOCK, 1997), um período mais longo de EPOC resultará em um aumento no gasto de energia (QUINN et al., 1994), o que deve gerar perda de peso;

$>$ Demonstrou-se que exercícios de alta intensidade realizados por certo período de tempo aumentam a taxa metabólica de repouso (TMR) (BØRSHEIM; BAHR, 2003; $\underline{\text { TREUTH }}$ et al., 1996), o que pode ser resultado de um aumento na massa muscular associado a essa forma de exercício (KING et al., 2001). Consequentemente, o exercício intervalado (que incorpora um componente de exercícios de alta intensidade), pode também resultar em um aumento na TMR, se praticado regularmente por certo período de tempo, com um aumento simultâneo no gasto de energia e na perda de peso (KING et al., 2001). De acordo com Quinn et al. (1994), o metabolismo elevado é essencial para uma perda de peso bem-sucedida, já que facilita a perda de peso mesmo se o indivíduo não estiver se exercitando;

$>$ A população em geral acredita que quando se quer perder peso, o exercício deve ser realizado em baixa intensidade, já que uma maior porcentagem de gordura é oxidada. Apesar dos exercícios de alta intensidade utilizar uma porcentagem mais baixa de gordura e uma porcentagem mais alta de oxidação de carboidratos para gerar energia, em comparação aos exercícios de baixa intensidade, as taxas absolutas de oxidação de gordura são similares (HANSEN et al., 2005; TALANIAN et al., 2007). A utilização de substrato não está limitada apenas à sessão de exercício, já que o exercício pode alterar a oxidação dos nutrientes e o metabolismo depois que a sessão já tiver sido realizada. Mesmo se os efeitos do exercício forem de baixos a moderados, a fase pós-exercício constitui em um período mais longo, e pode resultar em 
um efeito cumulativo substancial (HANSEN et al., 2005). De acordo com King et al. (2001), o número de calorias de gordura usadas durante e depois dos exercícios por certo período de tempo é, na verdade, maior durante exercícios de alta intensidade do que nos de baixa intensidade. Além disso, após exercícios de alta intensidade o metabolismo da gordura pode ser ainda mais alto, se for utilizado para repor as reservas reduzidas de glicogênio.

\section{Conclusão}

Pelas evidências reportadas nesta revisão, pode-se concluir que o exercício intervalado demonstrou resultados positivos na capacidade aeróbia tanto em atletas como em pessoas não atletas, melhorou também indicadores de composição corporal, como diminuição da gordura corporal e aumento da massa magra, e demonstrou ser eficiente também nos poucos estudos realizados com a população obesa. Além disso, é recomendado que ao se treinar de forma intervalada seja feita uma recuperação ativa durante os intervalos, pois além de atingir e manter $\circ \mathrm{VO}_{2 m a ́ x}$, estimula a remoção de lactato. Ademais, ao prescrever um treinamento intervalado para população obesa cuidados devem ser tomados no que diz respeito à intensidade e à recuperação do exercício.

\section{Referências}

ADENIRAN, S.A.; TORIOLA, A.L. Effects of continuous and interval running programmes on aerobic and anaerobic capacities in schoolgirls aged 13 to 17 years. The Journal of Sports Medicine and Physical Fitness, Torino, vol. 28, n. 3, p. 260-266, 1988. Disponível em: www.ncbi.nlm.nih.gov/pubmed/3068418. Acesso em: 10 Jun 2009.

AHMAIDI, S.; MASSE-BIRON, J.; ADAM, B.; CHOQUET, D.; FREVILLE, M.; LIBERT, J.P. et al. Effects of interval training at the ventilatory threshold on clinical and cardiorespiratory responses in elderly humans. European Journal of Applied Physiology and Occupational Physiology, Berlin, vol. 78, n. 2, p. 170-176. 1998. Disponível em:

http://dx.doi.org/10.1007/s004210050403. Acesso em: 05 Jun 2009.

AMATI, F.; DUBÉ, J.J.; SHAY, C.; GOODPASTER, B.H. Separate and combined effects of exercise training and weight loss on exercise efficiency and substrate oxidation. Journal of applied physiology, Bethesda, vol.105, n. 3, p. 825-831, 2008. Disponível em: http://dx.doi.org/10.1152/japplphysiol.90384.2008 . Acesso em: 05 jun 2009.
BILLAT, L.V. Interval training for performance: a scientific and empirical practice. Special recommendations for middle and long-distance running. Part I: aerobic interval training. Sports Medicine, Auckland, vol. 31, n. 1, p. 13-31, 2001. Disponível em:

http://web.ebscohost.com/ehost/pdf?vid=3\&hid=10 8\&sid=60c6e43b-7595-4c1c-836ec1c8632798a7\%40sessionmgr104 . Acesso em: 20 abr 2009.

BILLAT, V.L.; SLAWINKSI, J.; BOCQUET, V.; CHASSAING, P.; DEMARLE, A.; KORALSZTEIN, J.P. Very short (15s-15s) interval-training around the critical velocity allows middle-aged runners to maintain VO2 max for 14 minutes. International Journal of Sports Medicine, Stuttgart, vol. 22, n. 3, p. 201-208, 2001. Disponível em: http://dx.doi.org/10.1055/s-2001-16389 . Acesso em: 03 Jun 2009.

BILLAT, V.L.; SLAWINSKI, J.; BOCQUET, V.; DEMARLE, A.; LAFITTE, L.; CHASSAING, P. et al. Intermittent runs at the velocity associated with maximal oxygen uptake enables subjects to remain at maximal oxygen uptake for a longer time than intense but submaximal runs. European Journal of Applied Physiology, Berlin, vol. 81, n. 3, p. 188-196, 2000. Disponível em: http://dx.doi.org/10.1007/s004210050029 . Acesso em: 03 Jun 2009.

BØRSHEIM, E; BAHR, R. Effect of exercise intensity, duration and mode on post-exercise oxygen consumption. Sports Medicine,

Auckland, vol. 33, n. 14, p. 1037-1060, 2003.

Disponível em

http://web.ebscohost.com/ehost/detail?vid=1\&hid= 102\&sid=c293907a-dd2e-497f-89e5-

5be3a9ea0dea\%40sessionmgr109\&bdata $=$ JnNpd GU9ZWhvc3QtbGl2ZQ\%3d\%3d\#db=s3h\&jid=C5 $\mathrm{H} \# \mathrm{db}=\mathrm{s} 3 \mathrm{~h} \& \mathrm{jid}=\mathrm{C} 5 \mathrm{H} \# \mathrm{db}=\mathrm{s} 3 \mathrm{~h} \& \mathrm{jid}=\mathrm{C} 5 \mathrm{H} \# \mathrm{db}=\mathrm{s} 3 \mathrm{~h} \& \mathrm{ji}$ d=C5H . Acesso em: 05 Jun 2009.

DUNCAN, J.J.; GORDON, N.F.; SCOTT, C.B. Women walking for health and fitness. How much is enough? JAMA: The Journal of the American Medical Association, Chicago, vol. 266, n. 23, p. 3295-3299, 1991. Disponível em: http://jama.amaassn.org.w10049.dotlib.com.br/content/vol266/iss ue23/index.dtl. Acesso em: 11 Jun 2009.

FRANCH, J.; MADSEN, K.; DJURHUUS, M.S.; PEDERSEN, P.K. Improved running economy following intensified training correlates with reduced ventilatory demands. Medicine and Science in Sports and Exercise, Madison, vol. 30, n. 8, p. 1250-1256, 1998. Disponível em: http://vnweb.hwwilsonweb.com/hww/results/getRe sults.jhtml? DARGS=/hww/results/results commo n.jhtml.26 . Acesso em: 05 Jun 2009. 
GEORGIOU, D.; CHEN, Y.; APPADOO, S.; BELARDINELLI, R.; GREENE, R.; PARIDES, M.K. et al. Cost-effectiveness analysis of longterm moderate exercise training in chronic heart failure. The American Journal of Cardiology, New York, vol. 87, n. 8, p. 984-988, A4, 2001. Disponível em: http://dx.doi.org/10.1016/S00029149(01)01434-5. Acesso em: 03 Jun 2009.

HANSEN, K.; SHRIVER, T.; SCHOELLER, D. The effects of exercise on the storage and oxidation of dietary fat. Sports Medicine, Auckland, vol. 35, n. 5, p. 363-373, 2005. Disponível em: http://web.ebscohost.com/ehost/results?vid=2\&hid $=102 \&$ sid=c293907a-dd2e-497f-89e55be3a9ea0dea\%40sessionmgr109\&bquery $=\left(\mathrm{JN}_{+}\right.$ \%22Sports+Medicine\%22+and+DT+20050301)\&b data=JmRiPXMzaCZ0eXBIPTEmc2I0ZT1laG9zdC 1saXZl . Acesso em: 27 abr 2009.

HARDMAN, A.E. Issues of fractionization of exercise (short vs long bouts). Medicine and Science in Sports and Exercise, Madison, vol. 33, n. 6, p. S421-S427, 2001. Disponível em: http://journals.lww.com/acsm-

$\underline{\text { msse/pages/articleviewer.asp }}$ ? year=2001\&issue $=06001 \&$ article $=00010$ \&type $=$ abstract . Acesso em: 10 Jun 2009.

HUNTER, G.R.; WEINSIER, R.L.; BAMMAN, M.M.; LARSON, D.E. A role for high intensity exercise on energy balance and weight control. International Journal of Obesity, Hampshire, vol. 22, n. 6, p. 489-493, 1998. Disponível em: http://www.nature.com/ijo/journal/v22/n6/pdf/0800 629a.pdf . Acesso em: 11 Jun 2009.

KAMINSKY, L.A.; WHALEY, M.H. Effect of interval type exercise on excess post-exercise oxygen consumption (EPOC) in obese and normal-weight women. Medicine in Exercise, Nutrition and Health, 2, 106-111, 1993.

KING, J.; BROEDER, C.; BROWDER, K.; PANTON, L. A comparison of interval vs. Steadystate exercise on substrate utilization in overweight women. Medicine and Science in Sports and Exercise, Madison, vol. 34, n. 5, p. S130, 2002. Disponível em:

http://journals.Iww.com/acsmmsse/Citation/2002/05001/A Comparison of Inte rval Vs Steady State Exercise.726.aspx. Acesso em: 11 Jun 2009.

KING, J.; PANTON, L.; BROEDER, C.; BROWDER, K.; QUINDRY, J.; RHEA, L. A comparison of high intensity vs. Low intensity exercise on body composition in overweight women. Medicine and Science in Sports and Exercise, Madison, vol. 33, n. 5, p. S228, 2001. Disponível em: http://journals.lww.com/acsmmsse/Citation/2001/05001/A Comparison of Hig h Intensity Vs Low Intensity.1285.aspx . Acesso em: 09 Jun 2009.

MAKRIDES, L.; HEIGENHAUSER, G.J.; JONES, N.L. High-intensity endurance training in 20- to 30and 60- to 70-yr-old healthy men. Journal of applied physiology, Bethesda, vol. 69, n. 5, p. 1792-1798, 1990. Disponível em:

http://jap.physiology.org/cgi/content/abstract/69/5/ 1792. Acesso em: 30 abr 2009.

MCARDLE, W.; KATCH, F.I.; KATCH, V.L. Fisiologia do Exercício: Energia, Nutrição e Desempenho Humano. (5 ed.). Rio de Janeiro: Guanabara Koogan, 2003.

MEYER, K. Exercise training in heart failure: recommendations based on current research. Medicine and Science in Sports and Exercise, Madison, vol. 33, n. 4, p. 525-31, 2001. Disponível em:

http://vnweb.hwwilsonweb.com/hww/results/results common.jhtml;hwwilsonid=20ISUWAW5EBTRQ A3DIMSFF4ADUNGIIV0 . Acesso em: 27 abr 2009.

MILLET, G.P.; CANDAU, R.; FATTORI, P.; BIGNET, F.; VARRAY, A. VO2 responses to different intermittent runs at velocity associated with VO2max. Canadian Journal of Applied Physiology, Champaign, vol. 28, n. 3, p. 410-423, 2003. Disponível em:

http://web.ebscohost.com/ehost/detail?vid=3\&hid= 107\&sid=2e97a228-16f0-45ad-91d1-

20034d71ea5f\%40sessionmgr102\&bdata $=$ JnNpd GU9ZWhvc3QtbGl2ZQ\%3d\%3d\#db=s3h\&AN=SP HS-892308 Acesso em: 13 Jun 2009.

MOREIRA, P.B.B. Treinamento Aeróbico Intervalado na Insuficiência Cardíaca. Revista do Derc: Órgão oficial do Departamento de Ergometria, Reabilitação Cardíaca e Cardiologia Desportiva da SOCERJ, Rio de Janeiro, vol. 9, n. 35, p. 11-12, 2008. Disponível em: http://departamentos.cardiol.br/sbcderc/revista/2008/43/pdf/Rev43-p11-12.pdf . Acesso em: 03 Jun 2009.

O'DONOVAN, G.; OWEN, A.; BIRD, S.R.; KEARNEY, E.M.; NEVILL, A.M.; JONES, D.W. et al. Changes in cardiorespiratory fitness and coronary heart disease risk factors following $24 \mathrm{wk}$ of moderate- or high-intensity exercise of equal energy cost. Journal of applied physiology, Bethesda, vol. 98, n. 5, p. 1619-1625, 2005. Disponível em: http://dx.doi.org/10.1152/japplphysiol.01310.2004 . Acesso em: 05 Jun 2009.

PERRY, A.C.; TAPP, J.; WEEKS, L. The effects of interval aerobic training on plasma lipid fractions of male and post-menopausal sedentary faculty. The Journal of Sports Medicine and 
Physical Fitness, Torino, vol. 26, n. 2, p. 186193, 1986. Disponível em:

http://www.ncbi.nlm.nih.gov/pubmed/3747486 .

Acesso em: 10 Jun 2009.

QUINN, T.J.; VROMAN, N.B.; KERTZER, R. Postexercise oxygen consumption in trained females: effect of exercise duration. Medicine and Science in Sports and Exercise, Madison, vol. 26, n. 7, p. 908-913, 1994. Disponível em: http://vnweb.hwwilsonweb.com/hww/results/getRe sults.jhtml? DARGS=/hww/results/results commo n.jhtml.26 . Acesso em: 06 Jun 2009.

SHORT, K.R.; SEDLOCK, D.A. Excess postexercise oxygen consumption and recovery rate in trained and untrained subjects. Journal of applied physiology, Bethesda, vol. 83, n. 1, p. 153-159, 1997. Disponível em:

http://iap.physiology.org/cgi/content/full/83/1/153 . Acesso em: 10 Jun 2009.

SÖKMEN, B.; BEAM, W.; WITCHEY, R.; ADAMS, G. Effect of interval versus continuous training on aerobic and anaerobic variables. Medicine Medicine and Science in Sports and Exercise, Madison, vol. 34, n. 5, S. 91, 2002. Disponível em: http://journals.Iww.com/acsmmsse/Citation/2002/05001/Effect of Interval Vers us Continuous Training on.509.aspx . Acesso em: 30 abr 2009.

TALANIAN, J.L.; GALLOWAY, S.D.; HEIGENHAUSER, G.J.; BONEN, A.; SPRIET, L.L. Two weeks of high-intensity aerobic interval training increases the capacity for fat oxidation during exercise in women. Journal of Applied Physiology (Bethesda, Md.: 1985), Bethesda, vol. 102, n. 4, p. 1439-1447, 2007. Disponível em: http://dx.doi.org/10.1152/japplphysiol.01098.2006. Acesso em: 03 Jun 2009.

TJØNNA, A.E.; STØLEN, T.O.; BYE, A.; VOLDEN, M.; SLØRDAHL, S.A.; ODEGÅRD, R. et al. Aerobic interval training reduces cardiovascular risk factors more than a multitreatment approach in overweight adolescents. Clinical science, Londres, vol. 116, n. 4, p. 317-326, 2009. Disponível em: http://www.clinsci.org/cs/116/0317/cs1160317.htm . Acesso em: 29 Jun 2009.

TREMBLAY, A.; SIMONEAU, J.A.; BOUCHARD, C. Impact of exercise intensity on body fatness and skeletal muscle metabolism. Metabolism: clinical and experimental, New York, vol. 43, n. 7, p. 814-818, 1994. Disponível em: http://dx.doi.org/10.1016/0026-0495(94)90259-3 . Acesso em: 03 Jun 2009.

TREUTH, M.S.; HUNTER, G.R.; WILLIAMS, M. Effects of exercise intensity on 24-h energy expenditure and substrate oxidation. Medicine and Science in Sports and Exercise, Madison, vol. 28, n. 9, p. 1138-1143, 1996. Disponível em: http://vnweb.hwwilsonweb.com/hww/results/getRe sults.jhtml? DARGS=/hww/results/results commo n.jhtml.21 . Acesso em: 11 Jun 2009.

WILLARDSON, J.M.; EMMETT, J.; OLIVER, J.A.; BRESSEL, E. Effect of short-term failure versus nonfailure training on lower body muscular endurance. International Journal of Sports Physiology and Performance, Champaign, vol. 3, n. 3, p. 279-293, 2008. Disponível em: http://web.ebscohost.com/ehost/detail? vid=3\&hid= 102\&sid=ea6a4810-8c69-499a-9463b884dd5671e1\%40sessionmgr104\&bdata=JnNpd GU9ZWhvc3QtbG|2ZQ\%3d\%3d\#db=s3h\&AN=34 632961\#db=s3h\&AN=34632961. Acesso em: 14 Jun 2009.

\section{Endereço:}

Diego Augusto Santos Silva

Av. Gonçalo Rollemberg Leite, 1960/1201

Cond. Alphaville I, Edf. Gama, Suissa

Aracaju SE Brasil

49050-370

e-mail:diegoaugustoss@yahoo.com.br

Recebido em: 04 de julho de 2009.

Aceito em: 14 de dezembro de 2009.

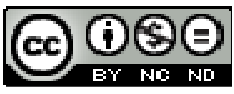

Motriz. Revista de Educação Física. UNESP, Rio Claro, SP, Brasil - elSSN: 1980-6574 - está licenciada sob Licenca Creative Commons 